\title{
Dynamic thiol-disulphide homeostasis in grade 3-4 gonarthrosis
}

\author{
Kenan Güvenç ${ }^{1} \odot$, Şahap Cenk Altun ${ }^{2} \odot$, Merve Ergin $^{3} \odot$, Özcan Erel ${ }^{4} \odot$, Faik $^{\text {İlik }}{ }^{5} \odot$ \\ ${ }^{1}$ Department of Orthopedics, Mersin University School of Medicine, Mersin, Turkey \\ ${ }^{2}$ Department of Orthopedics, Ankara Occupational Diseasses Hospital, Ankara, Turkey \\ ${ }^{3}$ Department of Biochemistry, Gaziantep State Hospital, Gaziantep, Turkey \\ ${ }^{4}$ Department of Biochemistry, Ankara Atatürk Training and Research Hospital, Ankara, Turkey \\ ${ }^{5}$ Department of Neurology, Karatay University School of Medicine, Konya, Turkey
}

DOI: $10.18621 /$ eurj.414367

\begin{abstract}
Objectives: We aimed to determine thiol-disulphide homeostasis, which plays a vital role and to investigate the relationship among homeostatic parameters and disease.

Methods: In this prospective study, we enrolled 38 patients with osteoarthritis (31 females and 7 males) and 38 healthy controls (30 females, 8 males volunteers). Diagnosis of osteoarthritis was made according to the American College of Rheumatology Criteria. The severity of osteoarthritis was assessed and classified according to the Kellgren-Lawrence grading scale.

Results: The mean age was 63.8 (range; 53-74) years in the osteoarthritis group and 65.6 (range; 55-75) years in the control group. There were no significant differences between the patients and controls in respect to age, gender and body mass index $(p>0.05)$. Serum albumin $(p=0.605)$ and total protein levels $(p=0.605)$ between patients and controls were similar. In the osteoarthritis group disulphide/ native thiol percent ratios and disulphide/ total thiol percent ratios were found to be statistically higher ( $p=0.002$ and $p=0.002$; respectively) and native/ total thiol percent ratios were significantly lower than that of the control group $(p=0.002)$.

Conclusions: Thiol-disulphide homeostasis is weakened in osteoarthritis, and the balance shifts to the disulphide bond formation side. Substitution of thiol deficiency and correction of thioldisulphide imbalance may be beneficial in the managing treatment of the disease. Further studies may be needed for evaluating articular fluid thiol-disulphide homeostasis.
\end{abstract}

Keywords: Oxidative stress, osteoarthritis, thiol-disulphide homeostasis

Received: April 11, 2018; Accepted: September 26, 2018; Published Online: June 30, 2019

$\mathrm{O}$ steoarthritis is a chronic, progressive disorder of thesynovial joints, characterized by focal loss of cartilage and changes in subchondral and marginal bone, synovium, and peri-articular structures [1]. Osteoarthritis of the knee is a relatively common condition that affects approximately $10 \%$ of the general population above the age of 55 years [2]. Radiographic appearance and clinical features are still often used for diagnosis of the disease. However, the etiology of osteoarthritis is not fully understood, although mechanical, biochemical, and genetic factors are accepted to play roles $[3,4]$.

One possible cause of osteoarthritis is oxidative stress. There is some evidence of the relationship be-

Address for correspondence: Kenan Güvenç, MD., Mersin University School of Medicine, Department Orthopedics, Mersin, Turkey E-mail: guvenckenan@hotmail.com 
tween knee osteoarthritis and oxidative stress $[3,5,6]$. The levels of pro-inflammatory mediators, such as reactive oxygen species (ROS), are elevated in osteoarthritis $[7,8]$. Thus, the increased levels of these reactive species with oxidative activity mediate the effects of many pro-inflammatory cytokines, such as interleukin (IL)-1 and tumor necrosis factor (TNF)- $\alpha$ [7-9]. IL-1 and TNF- $\alpha$ may play a crucial role in cartilage matrix degradation by stimulating matrix metalloproteinase (MMP) expression in patients with osteoarthritis $[8,9]$.

It is known that free radicals cause oxidation of $\mathrm{SH}$ groups in sulfur-containing amino acids of proteins and this is the earliest observable signs of protein oxidation [10]. To protect cells against oxidative stress, certain low molecular weight antioxidant molecules, either water-soluble (e.g., ascorbic acid) or lipid-soluble (e.g., vitamin E), are present in extracellular fluids [11]. Thiols are in interaction with almost all physiological oxidants. And they are mentioned as essential antioxidant buffers. Thiols, also known as mercaptans, which consist of a sulfur atom and a hydrogen atom bound to a carbon atom, are functional sulfhydryl groups [12]. A very large part of the blood plasma thiol pools consist mainly of albumin and other proteins such as glutathione, thioredoxin, cysteine and homocysteine [13]. Thiol groups of proteins are oxidized by oxygen molecules present in the medium and are reversibly converted to disulphide bonds. Formed disulphide bonds can be reduced to thiol groups again. Thus the thiol-disulphide balance is maintained [14]. Dynamic thiol-disulphide homeostasis plays a critical role in antioxidant defense, detoxification, apoptosis, regulation of enzyme activity, transcription and cellular signal transduction mechanisms $[15,16]$. Only a single side of this double-sided balance has been measured since 1979 [17]. Both variable levels are measured one by one and cumulatively with a novel and automated method [18].

We aimed to determine thiol-disulphide homeostasis, which plays a vital roleoa and to investigate the relationship among homeostatic parameters and disease.

\section{METHODS}

In this prospective study, we enrolled 38 patients with osteoarthritis ( 31 females and 7 males) and 38 healthy controls (30 females, 8 males volunteers). Diagnosis of osteoarthritis was made according to the American College of Rheumatology Criteria [19]. The severity of osteoarthritis was assessed and classified according to the Kellgren-Lawrence grading scale [20]. Grade 0 was accepted as normal, grade 1 as possible osteophytes only, grade 2 as absolute osteophytes and possible joint space narrowing, grade 3 as moderate osteophytes and/or absolute joint space narrowing, and grade 4 as large osteophytes, severe joint space narrowing, and/or bony sclerosis. All patients had grade III-IV knee osteoarthritis according to the radiological classification and clinical findings. Exclusion criteria included use of supplemental vitamins, smoking, diabetes mellitus, coronary artery disease, acute/chronic liver diseases, inflammatory rheumatic disease, clinically unstable medical illness, or the use of any medication within 4 weeks prior to initiation of the study.

All subjects were informed. Written consents were obtained and the study was approved by the local ethics committee. Patient and healthy groups were matched in terms of osteoarthritis grade and age.

Tablo1. Demographic characteristics of patients and controls

\begin{tabular}{lccc}
\hline & Osteoarthritis group & Control group & $p$ value \\
\hline $\begin{array}{l}\text { Age (years) } \\
\text { Mean (range) }\end{array}$ & $63.8(53-74)$ & $65.6(55-75)$ & 0.759 \\
$\begin{array}{l}\text { Gender, } \mathbf{n} \\
(\text { male } / \text { female) }\end{array}$ & $7 / 31$ & $8 / 30$ & $27.30(24.8-32.63)$ \\
BMI (kg/m $\left.{ }^{2}\right)$ & $26.74(25.5-31.75)$ & & \\
Mean (range) & & &
\end{tabular}


Venous blood samples were collected from the subjects and centrifuged at $2300 \mathrm{x} g$ for $10 \mathrm{~min}$. Serum samples were separated and stored at $-80{ }^{\circ} \mathrm{C}$ until analysis. Serum thiol -disulphide homeostasis was determined with a recently developed a novel and automatic measurement method by using an automated clinical chemistry analyser (Roche, cobas 501, Mannheim, Germany) [18]. Native thiol (-SH) and total thiol (-SH $+-\mathrm{S}-\mathrm{S}-)$ were measured directly, and -S-S-/-SH, -S-S-/-SH + -S-S-, -SH/-SH + -S-Sresults were obtained with calculation.

\section{Statistical Analysis}

All analyses were conducted using the SPSS software (version 22; IBM SPSS Inc., Chicago, IL, USA). The normality of distributions was evaluated using the one-sample Kolmogorov - Smirnov test, revealing a uniform distribution. Mann Whitney $U$ test was used to analyze the numerical variables. $p$-values of less than 0.05 were regarded as significant.

\section{RESULTS}

The mean age was 63.8 (range; 53-74) years in the osteoarthritis group and 65.6 (range; 55-75) years in the control group. Demographic characteristics of patients with knee osteoarthritis and controls are shown in Table 1. There were no significant differences between the patients and controls in respect to age, gender and body mass index (BMI). Serum albumin and total protein levels of the between patients and controls were similar $(p=0.605$ and $p=$ 0.652 ; respectively).

There is no difference in native thiol levels and total thiol levels between the groups $(p=0.06$ and $p=$
0.07 ; respectively ). In addition there is no diffference in disulphide values between the groups $(p=0.07)$. In the osteoarthritis group disulphide/ native thiol percent ratios and disulphide/ total thiol percent ratios were found to be statistically higher $(p=0.002$ and $p=$ 0.002 ; respectively) and native/ total thiol percent ratios were significantly lower than that of the control group $(p=0.002)$ (Table 2$)$.

\section{DISCUSSION}

Thiol groups have a significant role in the cell by minimizing the toxic effects of oxygenactivation processes. Fundamentally sulfhydryl groups are associated with proteins. So, when thiol levels decreases in serum its antioxidant power will decrease too. Because reactive species organized near the sides of their formation, increases in the expression of protein levels of thiol-disulphide will protect the tissular oxidative damage and cannot prevent the oxidation of thiol groups in serum [10-14].

Dynamic thiol-disulphide homeostasis has a critical role in the organism. Changes in the thioldisulphide balance serve as components for antioxidant protection, detoxification, regulation of enzymatic activity and cellular signaling mechanisms $[16,21]$. Changes in thioldisulphide homeostasis have been associated with various diseases such as diabetes mellitus, cancer, chronic kidney disease, liver disorders and chronic obstructive pulmonary disease [22-24].

Osteoarthritis is a process of progressive deterioration of articular cartilage and formation of osteophyte at the joint surface. Osteoarthritis is often associated with significant disability and an impaired

Table 2. Thiol-disulphide profiles of subjects

\begin{tabular}{lccc}
\hline \multicolumn{1}{c}{ Parameter } & Osteoarthritis group & Control group & $\boldsymbol{p}$ value \\
\hline -SH $(\mu \mathrm{mol} / \mathrm{L})$ & $375.07 \pm 34.512$ & $407.66 \pm 48.99$ & 0.603 \\
-S-S- $(\mu \mathrm{mol} / \mathrm{L})$ & $15.50 \pm 4.507$ & $18.76 \pm 4.59$ & $\mathbf{0 . 0 0 3}$ \\
-SH + -S-S $(\mu \mathrm{mol} / \mathrm{L})$ & $405.992 \pm 37.493$ & $407.66 \pm 48.99$ & 0.0712 \\
-S-S- /-SH + -S-S- $(\%)$ & $3.806 \pm 1.030$ & $4.61 \pm 1.090$ & $\mathbf{0 . 0 0 2}$ \\
-SH/-SH + -S-S- $(\%)$ & $92.407 \pm 2.006$ & $90.80 \pm 2.21$ & $\mathbf{0 . 0 0 2}$ \\
-S-S- / -SH $(\%)$ & $4.145 \pm 1.217$ & $5.11 \pm 1.093$ & $\mathbf{0 . 0 0 2}$ \\
\hline
\end{tabular}

$-\mathrm{SH}=$ native thiol, $-\mathrm{S}-\mathrm{S}-=$ disulphide, $(-\mathrm{SH}+-\mathrm{S}-\mathrm{S}-=$ total thiol 
quality of life. Pathologically, the disease is characterized by fissuring and focal erosive cartilage lesions, as well as cartilage loss and destruction [5]. Oxidative stress leads to increased risk for osteoarthritis but the precise mechanism remains unclear. Studies suggested that oxidative stress causes chondrocyte senescence and cartilage ageing [24, 25].

Soran et al. [5] and Altindag et al. [6] found that oxidative stress extremely increased in osteoarthritis, which may be responsible for the ethiopathogenesis of the disease). In our study, we observed decreased antioxidant parameters in subjects with knee osteoarthritis compared to the controls. These results confirmed the presence of oxidative stress.

\section{CONCLUSION}

In conclusion, thiol-disulphide homeostasis is weakened in osteoarthritis, and the balance shifts to the disulphide bond formation side. Substitution of thiol deficiency and correction of thioldisulphide imbalance may be beneficial in the managing treatment of the disease. Further studies may be nedeed for evaluating articular fluid thiol-disulphide homeostasis.

\section{Conflict of interest}

The author disclosed no conflict of interest during the preparation or publication of this manuscript.

\section{Financing}

The author disclosed that they did not receive any grant during conduction or writing of this study.

\section{REFERENCES}

[1] Felson D. The epidemiology of osteoarthritis: prevalence and risk factors. In: Kuettner KE, Goldberg VM, eds. Osteoarthritic Disorders. Rosemont, IL: American Academy of Orthopaedic Surgeons; 1995:13-24.

[2] Petersson IF. Occurrence of osteoarthritis of the peripheral joints in European populations. Ann Rheum Dis 1996;55:659-61. [3] Ertürk C, Altay MA, Selek S, Koçyiğit A. Paraoxonase-1 activity and oxidative status in patients with knee osteoarthritis and their relationship with radiological and clinical parameters. Scand J Clin Lab Invest 2012;72:433-9.

[4] Brandt KD, Dieppe P, Radin EL. Commentary: Is it useful to subset "primary" osteoarthritis? A critique based on evidence regarding the etiopathogenesis of osteoarthritis. Semin Arthritis Rheum 2009;39:81-95.

[5] Soran N, Altindag O, Cakir H, Celik H, Demirkol A, Aksoy $\mathrm{N}$. Assessment of paraoxonase activities in patients with knee osteoarthritis. Redox Rep. 2008;13:194-8.

[6] Altindag O, Erel O, Aksoy N, Selek S, Celik H, Karaoglanoglu M. Increased oxidative stress and its relation with collagen metabolism in knee osteoarthritis. Rheumatol Int. 2007;27:339-44.

[7] Ziskoven C, Jäger M, Zilkens C, Bloch W, Brixius K, Krauspe R. Oxidative stress in secondary osteoarthritis: from cartilage destruction to clinical presentation? Orthop Rev (Pavia) 2010;2:e23.

[8] Davies CM, Guilak F, Weinberg JB, Fermor B. Reactive nitrogen and oxygen species in interleukin-1-mediated DNA damage associated with osteoarthritis. Osteoarthritis Cartilage 2008;16:624-30.

[9] Martel-Pelletier J, Pelletier JP. Is osteoarthritis a disease involving only cartilage or other articular tissues? Eklem Hastalik Cerrahisi 2010;21:2-14.

[10] Kayalı R, Çakatay U. [Basic mechanisms of protein oxidation]. Cerrahpaşa J Med 2004;35: 83-9. [Article in Turkish] [11] Ustundag Y, Huysal K, Kahvecioglu K, Demirci H, Yavuz $\mathrm{S}$, Sambel M, et al. Establishing reference values and evaluation of an in-house ferric reducing antioxidant power (FRAP) colorimetric assay in microplates. Eur Res J 2016;2:126-31.

[12] Sen CK, Packer L. Thiol homeostasis and supplements in physical exercise. Am J Clin Nutr 2000;72(2 Suppl.):653S-69S. [13] Turell L, Radi R, Alvarez B. The thiol pool in human plasma: the central contribution of albumin to redox processes. Free Radic Biol Med 2013;65:244-53.

[14] Jones DP, Liang Y. Measuring the poise of thiol/disulfide couples in vivo. Free Radic Biol Med 2009; 47:1329-38.

[15] Circu ML, Aw TY. Reactive oxygen species, cellular redox systems, and apoptosis. Free Radic Biol Med 2010;48:749-62.

[16] Biswas S, Chida AS, Rahman I. Redox modifications of protein-thiols: emerging roles in cell signaling. Biochem Pharmacol 2006;71:551-64.

[17] Ellman G, Lysko H. A precise method for the determination of whole blood and plasma sulfhydryl groups. Anal Biochem 1979;93:98-102.

[18] Erel O, Neselioglu S. A novel and automated assay for thiol/disulphide homeostasis. Clin Biochem 2014;47:326-32.

[19] Altman R, Asch E, Bloch D, Bole G, Borenstein D, Brandt $\mathrm{K}$, et al. Development of criteria for the classification and reporting of osteoarthritis. Classification of osteoarthritis of the knee. Diagnostic and therapeutic criteria committee of the American Rheumatism Association. Arthritis Rheum 1986;29:1039-49.

[20] Kellgren JH, Lawrence JS. Radiological assessment of osteoarthrosis. Ann Rheum Dis 1957;16:494-502.

[21] Rodrigues SD, Batista GB, Ingberman M, Pecoits-Filho R, Nakao LS. Plasma cysteine/cystine reduction potential correlates with plasma creatinine levels in chronic kidney disease. Blood Purif 2012;34:231-7.

[22] Prabhu A, Sarcar B, Kahali S, Yuan Z, Johnson JJ, Adam $\mathrm{KP}$, et al. Cysteine catabolism: a novel metabolic pathway 
contributing to glioblastoma growth. Cancer Res 2014;74:78796.

[23] Eroğlu O, Dindar Badem N, Baccioğlu A, Cömertpay E, Neșelioğlu S, Erel Ö. Significance of thiol/disulphide homeostasis and ischemia modified albumin levels in chronic obstructive pulmonary disease. Eur Res J 2019;5:250-7.

[24] Yudoh K, Nguyen T, Nakamura H, Hongo-Masuko K, Kato $\mathrm{T}$, Nishioka K. Potential involvement of oxidative stress in cartilage senescence and development of osteoarthritis: oxidative stress induces chondrocyte telomere instability and downregulation of chondrocyte function. Arthritis Res Ther 2005; 7:R380-91.

[25] Martin JA, Brown TD, Heiner AD, Buckwalter JA. Chondrocyte senescence, joint loading and osteoarthritis. Clin Orthop Relat Res 2004;427 Suppl:S96-103. 